\title{
Optimizing MR imaging for intraoperative image guidance in sellar pathologies
}

\author{
Alexander Micko ${ }^{1}$ Arthur Hosmann ${ }^{1} \cdot$ Wolfgang Marik $^{2} \cdot$ Sophie Bartsch $^{2} \cdot$ Michael Weber $^{2} \cdot$ Engelbert Knosp $^{1}$. \\ Stefan Wolfsberger ${ }^{1}$
}

Published online: 13 March 2020

(c) The Author(s) 2020

\begin{abstract}
Purpose With the advancement of extended endonasal approaches, the ability to surgically reach parasellar tumor extensions increase. The aim of the study was to propose an optimized imaging protocol for surgical guidance in the cavernous sinus (CS) for proper visualization structures at risk.

Methods Prospective case control analysis of 20 consecutive pituitary adenoma patients scheduled for endoscopic transnasal surgery. Assessment of the capability of three different MRI sequences (MPRAGE, VIBE, CISS) by 4 investigators to correctly visualize sellar and parasellar structures. Invasiveness and position of the normal pituitary gland were compared with the intraoperative findings.

Results The consensus between the 4 examiners to achieve the same results for all modalities was 40\% for MPRAGE, 70\% for VIBE and 60\% for CISS sequences $(\mathrm{p}=0.155)$. A consensus of Knosp Grade per patient was $80 \%$ for MPRAGE, $100 \%$ for VIBE and 90\% for CISS (overall kappa 0.60). A higher Knosp Grade was found in MPRAGE sequences compared to the other sequences. Intraoperative status of invasiveness was correctly identified in 12/20 (60\%) with MPRAGE, 19/20 (95\%) with VIBE and 11/20 (55\%) with CISS sequences. The position of the normal pituitary gland was most frequent evaluable in $15 / 20(75 \%)$ and correctly identified in $12 / 15(80 \%)$ cases.

Conclusion Our data showed that VIBE sequences obtain the highest degree of consensus with intraoperative findings of invasiveness and position of the normal pituitary gland. VIBE sequences, due to their high spatial resolution and at the same time fast image acquisition could provide improved imaging for neuronavigation.
\end{abstract}

Keywords Pituitary adenoma $\cdot$ Neuronavigation $\cdot$ VIBE $\cdot$ CISS

\section{Introduction}

Pituitary adenomas are neuroendocrine tumors that show invasive growth into surrounding structures in up to $40 \%$ [1-5]. Especially parasellar invasiveness into cavernous sinus (CS) structures is still one of the most important prognostic factors for dismal outcome [6-9].

With the advancement of extended endonasal approaches (EEA), the ability to surgically reach parasellar tumor extensions increases. Hence, there is a demand for proper

Stefan Wolfsberger

stefan.wolfsberger@meduniwien.ac.at

1 Department of Neurosurgery, Medical University of Vienna, Waehringer Guertel 18-20, 1097 Vienna, Austria

2 Department of Biomedical Imaging and Image-guided Therapy, Medical University of Vienna, Vienna, Austria visualization of the neurovascular CS structures at risk such as cranial nerves and arteries and their relation to parasellar pituitary adenoma components.

The imaging modality of choice for soft tissue structures is magnetic resonance imaging (MRI). Currently, contrast enhanced T1-weighted MPRAGE MRI is widely used for delineation of pituitary gland and adenoma tissue as well as for the identification of the intracavernous internal carotid artery (ICA), because of its short acquisition time and isovoxel capability.

Neuronavigation systems, which by reconstruction of isovoxel image data provide the surgeon with multiplanar views for preoperative planning and intraoperative orientation, have found wide acceptance for use in endoscopic transsphenoidal surgery [10-20], especially for EEA. However, MPRAGE sequences only provide limited visualization 
detail of the adenoma extensions into and their relation to the neurovascular structures within the CS.

With advancements in MRI technology, sequences have been developed that can readily identify the tumor extension into the CS as well as the CS structures in a preoperative planning setting $[21,22]$. Two sequences have turned out to be the most useful:

Fat sat VIBE (volumetric interpolated breath-hold examination) images are fat-suppressed sequences that permit short acquisition times with high-resolution and therefore allow volumetric acquisition with an isotropic matrix. This allows high-resolution multiplanar reconstruction giving not only parenchymal but also vascular information at the same time through the application of contrast agent. VIBE sequences can therefore be used for high spatial resolution and improved soft tissue contrast imaging of the pituitary $[23,24]$.

CISS (constructive interference in steady state) sequences on the other hand provide better visualization of the cranial nerves and cisternal spaces due to the ability to accentuate T2-weighted values [25]. Especially by the usage of gadolinium based contrast agents, superior delineation of CS nerves in the intracavernous segment can be achieved [26]. CISS has thereby been shown to have a predictive value of postoperative visual outcomes and adenoma pathology in retrospective studies [27-29].

The aim of the study was to propose an optimized imaging protocol for surgical guidance in the CS by intraoperatively comparing tumor extension and neurovascular structures visualized on different MRI sequences with the concurrent surgical findings.

\section{Methods}

Prospective case control analysis of 20 consecutive pituitary adenoma patients scheduled for endoscopic transnasal surgery at our institution in 2019. We assessed the capability of three different MRI sequences (MPRAGE, VIBE, CISS) to correctly visualize cavernous sinus neurovascular structures and the grade of parasellar tumor extension compared with the intraoperative view.

The following parameters were evaluated: Parasellar tumor extension (according to the revised Knosp classification [5]), surgical invasiveness, identification and position of the normal pituitary gland, the optic chiasm, and the oculomotor nerve within the cavernous sinus.

Histopathological analysis according to the 2017 WHO classification of pituitary adenomas was performed as in the routine postoperative setting [30].

This study was approved by the hospital's ethics committee (EC Nr. 1549/2019) and was performed in accordance with the principles of the Declaration of Helsinki. Prior to enrollment into the trial, participants gave their written informed consent.

\section{Magnet resonance imaging parameters}

For this study, our routine preoperative MRI protocol for pituitary image guidance (MPRAGE, MRA — time of flight) was extended with VIBE and CISS sequences (additional 9 min scanning time). All examinations were performed on a 3T magnetic resonance scanner (3 T Vida, Siemens) using a 64-channel head and neck coil. Exclusion criteria were: claustrophobia, metal devices in or on the subject's body which contradict a $3 \mathrm{~T}$ examination. (Table 1)

MPRAGE: coronary slices, isovoxel imaging $(0.9 \mathrm{~mm}$ voxel size), TR: $2300 \mathrm{~ms}$, TE: $2.32 \mathrm{~ms}$, Matrix: 256, Flip angle: $8^{\circ}$; FoV: $240 \mathrm{~mm}$, slice thickness: $0.9 \mathrm{~mm}$, number of slices: 180 , acquisition time: 5:21 min;

VIBE: coronary slices, fat saturation, isovoxel imaging (0.7 mm voxel size), TR: $7.01 \mathrm{~ms}$, TE: $2.58 \mathrm{~ms}$, Matrix: 288, Flip angle: $15^{\circ}$; FoV: $210 \mathrm{~mm}$, slice thickness: $0.7 \mathrm{~mm}$, number of slices: 144 , acquisition time: $3: 17 \mathrm{~min}$;

CISS: coronary slices, $0.5 \mathrm{~mm}$ voxel size, TR: $8.33 \mathrm{~ms}$, TE: $3.89 \mathrm{~ms}$, Matrix: 256, Flip angle: 50 ; FoV: $140 \mathrm{~mm}$, slice thickness: $0.5 \mathrm{~mm}$, number of slices: 80 , acquisition time: 6:51 min.

Table 1 Patient characteristics

\begin{tabular}{lll}
\hline & Patient cohort & $\%$ \\
\cline { 2 - 3 } & $\mathrm{n}(\mathrm{IQR})$ & \\
\hline Number of patients & 20 & \\
Age (median, years) & $38(28-59)$ & 65 \\
Gender & & 35 \\
Female & 13 & \\
Male & 7 & \\
Primary/re-operation & $18 / 2$ & \\
Tumor size*, mm (median max. & $18.5(11-24)$ & \\
$\quad$ diameter) & & 45 \\
Micro-/macroadenoma & $4 / 16$ & 55 \\
Functional classification & & \\
Functional & 9 & 5 \\
Non-functional & 11 & 30 \\
WHO 2017 classification & & 5 \\
Somatotroph & 1 & 5 \\
Lactotroph & 6 & 30 \\
Thyrotroph & 1 & 20 \\
Corticotroph & 1 & 5 \\
Gonadotroph & 6 & \\
Null cell & 4 & \\
Plurihormonal & & \\
\hline
\end{tabular}

$I Q R$ interquartile range

*Mean diameter of MPRAGE/VIBE/CISS 


\section{Neuronavigation}

This study was based on our multimodality navigation protocol published in 2019 that includes computed tomography (CT) for solid bone structures and fine paranasal sinus structures [31], MRA-TOF for vascular anatomy, and standard T1-weighted CE MRI (MPRAGE) for pituitary and CS structures. The latter was always found insufficient to visualize CS structures in detail.

The ability of the three MRI sequences (MPRAGE, VIBE or CISS) to correctly visualize the anatomy of the $\mathrm{CS}$ as part of the multimodality MRI imaging protocol was assessed by four investigators, two neuroradiologists (S.B. and W.M.) and two neurosurgeons (A.H. and A.M.) blinded to the intraoperative results. (Fig. 1) The following parameters were assessed on neuronavigation systems (Stealth Station S7, Medtronic, USA): Identification and position of the normal pituitary gland, visibility of the oculomotor nerves, parasellar tumor extension (graded according to the revised Knosp classification [5]) and surgical invasiveness. Consensus results of investigators were then compared to the ground truth of intraoperative findings. (Fig. 2)

\section{Statistical evaluation}

The data are presented as median and interquartile range (IQR) for continuous variables and as frequencies for categorical variables. Agreement upon the 4 reviewers had to be at least $3 / 4$ to conclude a consensus.

The consensus for each assessed parameter was used to compare the MRI sequences (MPRAGE, VIBE or CISS) among each other. Additionally, the consensus of invasiveness and identification and position of the normal pituitary gland was correlated with the results of intraoperative findings. The probability of a consensus between the 4 examiners was carried out using the Cochran $\mathrm{Q}$ test. The agreement of the raters per examined modality and sequence was calculated using Fleiss Kappa.

A p-value $<0.05$ will be considered significant. For statistical analyses SPSS ${ }^{\circledR}$ version 24.0 software (SPSS Inc., Chicago, IL, USA) was used.
A

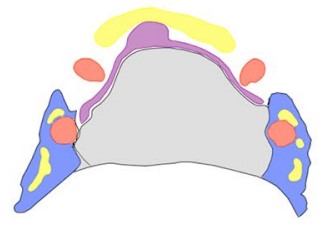

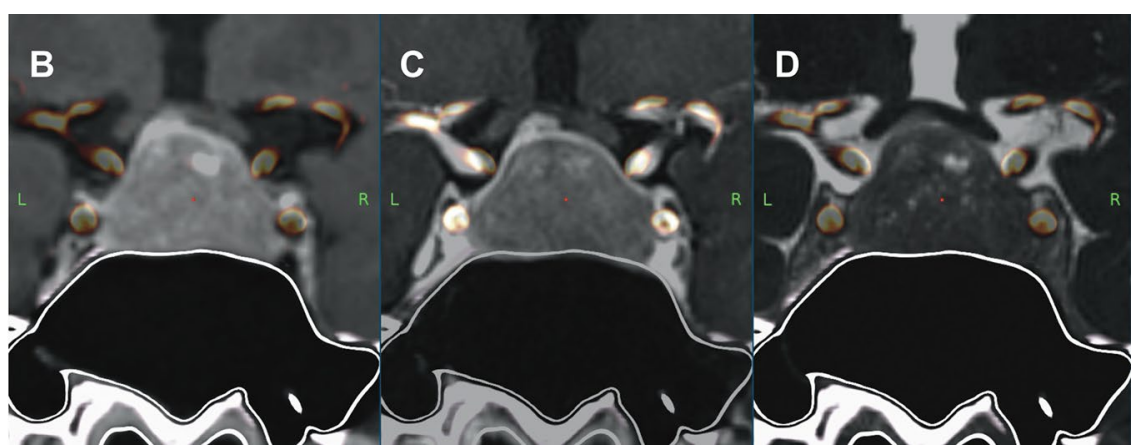

artery $($ ICA), yellow $=$ cranial nerves, blue $=$ cavernous sinus $(C S)$. $\mathbf{b}$ Multimodality imaging, MPRAGE as MR sequence. c Multimodality imaging, VIBE as MR sequence. d Multimodality imaging, CISS as MR sequence
Fig. 1 Multimodality Imaging, combination of MR, 2x CT (1 for solid bone structures, 1 for visualization of fine paranasal structures), MR angiogram (time-of-flight). a Schematic drawing: grey = pituitary adenoma, purple $=$ normal pituitary gland, red $=$ internal carotid

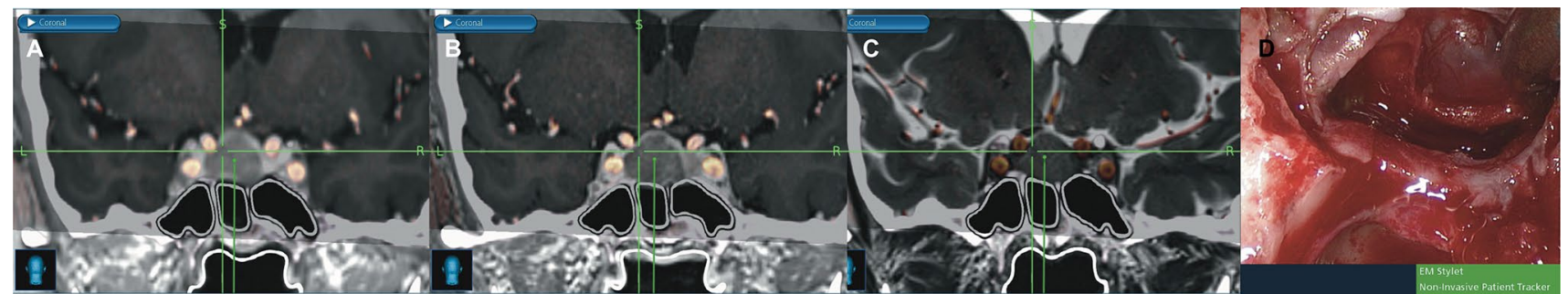

Fig. 2 Intraoperative Neuronavigation, correlation with anatomic structures. a Multimodality imaging, MPRAGE as MR sequence. b Multimodality imaging, VIBE as MR sequence. c Multimodality imaging, CISS as MR sequence. d Intraoperative image; an electro- magnetic stylet was placed in a suction device. Green line with dot.... planned trajectory at the center of the pituitary adenoma. Green crosshair... depiction of the location of the tip of the electromagnetic stylet within the suction device 


\section{Results}

\section{Patient characteristics}

Median age at the time of operation was 38 years (IQR 28-59). Functioning adenomas represented 9/20 (45\%) of patients, whereas $11 / 20(55 \%)$ were non-functioning adenomas. Within the study cohort $4 / 20(20 \%)$ were microadenomas and 16/20 (80\%) were macroadenomas. The maximal tumor diameter, calculated as a mean of MPRAGE/VIBE/ CISS sequences, was median $18.5 \mathrm{~mm}$ (IQR 11-24 mm). For further patient characteristics, see Table 1 .

\section{Rater consensus}

The consensus between the 4 examiners for all parameters assessed (grade of parasellar tumor extension according to the revised Knosp Classification; invasiveness; identification and position of the normal pituitary gland; visibility of the optic chiasm; visibility of the oculomotor nerves) was $40 \%$ for MPRAGE, $70 \%$ for VIBE and $60 \%$ for CISS sequences $(\mathrm{p}=0.155)$.

Only the position of the normal pituitary gland showed significant differences of examiners consensus between the MR sequences $(p=0.045)$, in all other examined parameters no significant difference was present.

Parasellar tumor extension as evaluated by the revised Knosp classification showed a complete consensus of examiners in 28/40 (70\%) (left and right CS compartment) independent of the examined MR sequence.

A consensus of Knosp grade per patient was $80 \%$ for MPRAGE, $100 \%$ for VIBE and $90 \%$ for CISS (overall kappa 0.60). A higher Knosp grade was found in MPRAGE sequences in 6 cases, compared to VIBE and CISS sequences. (Table 2)

Intraoperative invasiveness was found in 4/20 (20\%) of cases. A false positive result turned out in 3 MPRAGE, 1 VIBE and 3 CISS cases; a false negative result turned out in 2 CISS cases.

\section{Pituitary gland location}

The normal pituitary gland was found to be located in 13/20 (65\%) cases lateral and in 7/20 (35\%) cases superior to the tumor mass. A false lateral (instead of intraoperative superior) position was suspected in 2 MPRAGE, 3 of VIBE and 3 of CISS cases. A false superior (instead of intraoperative lateral) position was suspected in 2 CISS cases.

Cranial nerves: The optic chiasm by consensus could be detected in 13/17 (76\%) of MPRAGE, 16/18 (89\%) of VIBE and $17 / 19(89 \%)$ of CISS cases.

The oculomotor nerve within the cavernous sinus could be detected by consensus in 13/19 (68\%) of MPRAGE, $11 / 15(73 \%)$ of VIBE and 13/15 (87\%) CISS cases. (Table 3)

\section{Discussion}

The MR sequences most widely used for intraoperative guidance are conventional T1-weighted CE MPRAGE sequences [20,32]. However, newer MR sequences such as VIBE and CISS have been found to identify intra- and parasellar structures to a higher detail. According to our data of multimodality neuronavigation imaging, MPRAGE sequences tend to overestimate the parasellar extension of the tumor mass.
Table 2 Knosp Classification between the MR sequences (40 cavernous sinus compartments)

\begin{tabular}{lllllll}
\hline & Knosp 0 & Knosp 1 & Knosp 2 & Knosp 3A & Knosp 3B & Knosp 4 \\
\hline MPRAGE* & 18 & 8 & 5 & 1 & - & 4 \\
VIBE & 24 & 8 & 7 & - & - & 1 \\
CISS* & 21 & 7 & 2 & 6 & - & - \\
\hline
\end{tabular}

*...in 4 cavernous sinus compartments no consensus was reached

\begin{tabular}{|c|c|c|c|c|c|c|}
\hline & \multicolumn{2}{|l|}{ Invasiveness } & \multicolumn{2}{|c|}{ Normal gland position } & \multirow{2}{*}{$\begin{array}{l}\text { Optic chiasm detected } \\
\mathrm{n} / \mathrm{n}(\%)\end{array}$} & \multirow{2}{*}{$\begin{array}{l}\text { Oculomo- } \\
\text { tor nerve } \\
\text { detected } \\
\mathrm{n} / \mathrm{n}(\%)\end{array}$} \\
\hline & $\begin{array}{l}\text { Consensus } \\
\mathrm{n} / \mathrm{n}(\%)\end{array}$ & $\begin{array}{l}\text { Accuracy } \\
\mathrm{n} / \mathrm{n}(\%)\end{array}$ & $\begin{array}{l}\text { Consensus } \\
\mathrm{n} / \mathrm{n}(\%)\end{array}$ & $\begin{array}{l}\text { Accuracy } \\
\mathrm{n} / \mathrm{n}(\%)\end{array}$ & & \\
\hline MPRAGE & $15 / 20(75)$ & $12 / 20(60)$ & $8 / 20(40)$ & $6 / 20(30)$ & $13 / 17 *(76)$ & $13 / 19 *(68)$ \\
\hline VIBE & $20 / 20(100)$ & $19 / 20(95)$ & $15 / 20(75)$ & $12 / 20(60)$ & $16 / 18 *(89)$ & $11 / 15^{*}(73)$ \\
\hline CISS & $16 / 20(80)$ & $11 / 20(55)$ & $11 / 20(55)$ & $6 / 20(30)$ & $17 / 19 *(89)$ & $13 / 15^{*}(87)$ \\
\hline
\end{tabular}

*...in the missing cases no consensus was reached between examiners
Table 3 Evaluation of invasiveness, position of the pituitary gland, optic chiasm and oculomotor nerve 
VIBE sequences showed the highest degree of consensus with intraoperative findings of invasiveness and position of the normal pituitary gland. CISS sequences could identify the oculomotor nerve in most of the cases.

\section{MR sequences for visualization of sellar and parasellar structures}

Especially in cases of large tumors markedly displacing the optic chiasm, the normal gland tissue and neurovascular structures within the cavernous sinus, a proper correlation of intraoperative and neuronavigation images is crucial. Sequences that are able to establish high spatial resolution and therefore the possibility to better distinguish contrast difference have been investigated [22, 23]. VIBE sequences have been found to reach a high spatial resolution and therefore improve the visualization of soft tissue. CISS or FIESTA (Fast Imaging Employing Steady-state Acquisition, GE Healthcare equivalent of CISS) have a high fluid signal and high spatial resolution.

Xie et al. [33] first presented a case series of 7 patients where they used FIESTA sequences in the preoperative planning to approach sellar and parasellar lesions and found to properly visualize vascular and neural structures.

\section{Parasellar adenoma extension}

Knosp grading was found variable between the MR sequences. Our results of pituitary adenomas are in accordance with Lang et al. [21] that MPRAGE sequences have been found to overestimate the parasellar tumor extension. CISS sequences might misinterpret the parasellar extension as they are prone to artifacts [34]. In contrast to the results of Lang et al. we found VIBE sequences to correlate with the highest degree (95\%) to intraoperatively identified tumor invasiveness by direct endoscopic visualization. VIBE may facilitate the depiction of invasive tumor components through a higher grade of visualization of enhancing structures within the CS. Therefore, a distinction between pituitary adenoma tissue and CS structures may be more accurate in VIBE than in MPRAGE or CISS sequences.

Position of the pituitary gland: Preoperative localization of the normal pituitary gland is crucial to prevent inadvertent surgical damage and consecutive hormonal insufficiency. To define the position of the pituitary gland, Davis et al. compared conventional T1-weighted CE sequences versus VIBE sequences in 32 patients, and found in more than $50 \%$ of cases that investigators rated delineation of the gland more accurately with VIBE [23]. In our prospective analysis investigators reached the highest grade of consensus on VIBE sequences $(15 / 20)$ and the gland was correctly identified in $12 / 15$ cases ( $80 \%$ ). In comparison to conventional T1-weighted CE sequences (MPRAGE) a worse rate of consensus was reached (8/20, $6 / 8$ correctly identified) in CISS sequences (11/20 consensus), and the position of the gland could only be correctly identified in $6 / 11$ cases (54\%).

\section{Cranial nerves}

CISS sequences provide a good delineation of the optic pathway and cranial nerves relative to the tumor mass because of a low signal intensity and proper distinction of cerebrospinal fluid $[27,28]$. In case of tumor involvement, the visualization of cranial nerves within the CS with these sequences has been found to be depended which nerve is involved. Amemiya et al. were able to visualize the oculomotor nerve in all cases whereas the abducent nerve was only seen in half of their cases [35].

Although CISS sequences have a striking advantage in visualization of cranial nerves, the possible overestimation of parasellar invasive growth and disadvantage of failing to correctly delineate the position of remaining normal gland tissue are drawbacks for using primarily this sequence for neuronavigation. However, due to the possibility to mark the cranial nerves as region of interests, this information can be used in a multimodality imaging setting as an addition.

\section{Microadenomas}

Detection of microadenomas by VIBE sequences has been found to improve the detection from 60-80\% compared with conventional T1-weighted CE MRI sequences [36-39].

Examining previously MR-negative patients with Cushing's disease, Grober et al. [24] found that SGE (spoiledgradient echo 3D T1 imaging; GE Healthcare equivalent of VIBE) is superior to conventional T1-weighted CE MRI sequences to identify corticotroph microadenomas. From our series, we cannot confirm these results, as only $20 \%$ (4/20) were microadenomas and no corticotroph adenoma was included. However, we found VIBE sequences to better delineate the pituitary gland details.

\section{Limitations}

Due to the limited number of patients included in this prospective trial, no significant difference of investigators consensus between the three MR sequences could be reached. However, to the best of our knowledge this is the first study investigating MPRAGE/VIBE/CISS sequences in a multimodality neuronavigation setting.

Although VIBE sequences have been found to correlate with the highest degree with intraoperative findings such as invasiveness and position of the remaining pituitary gland, this sequence has a high susceptibility to motions causing artifacts. 


\section{Conclusions}

With the advancement of extended endonasal approaches the demand for proper visualization of sellar and parasellar structures at risk via neuronavigation images is necessary. In this prospective trial, our data showed that VIBE sequences obtain the highest degree of consensus with intraoperative findings of invasiveness and position of the normal pituitary gland. Therefore, VIBE sequences due to their high spatial resolution and at the same time fast image acquisition could provide improved preoperative images in an isovoxel protocol for neuronavigation.

However, additional CISS sequences could be necessary to identify the oculomotor nerve in pituitary adenomas that show an extensive parasellar growth.

Acknowledgements Open access funding provided by Medical University of Vienna.

Funding This research did not receive any specific grant from any funding agency in the public, commercial or not-for-profit sector.

\section{Compliance with ethical standards}

Conflict of interest The authors report no conflict of interest concerning the materials or methods used in this study or the findings specified in this paper. Stefan Wolfsberger is currently Educational Consultant of Medtronic Surgical Technologies.

Open Access This article is licensed under a Creative Commons Attribution 4.0 International License, which permits use, sharing, adaptation, distribution and reproduction in any medium or format, as long as you give appropriate credit to the original author(s) and the source, provide a link to the Creative Commons licence, and indicate if changes were made. The images or other third party material in this article are included in the article's Creative Commons licence, unless indicated otherwise in a credit line to the material. If material is not included in the article's Creative Commons licence and your intended use is not permitted by statutory regulation or exceeds the permitted use, you will need to obtain permission directly from the copyright holder. To view a copy of this licence, visit http://creativecommons.org/licenses/by/4.0/.

\section{References}

1. Fahlbusch R, Buchfelder M (1988) Transsphenoidal surgery of parasellar pituitary adenomas. Acta Neurochir (Wien) 92(1-4):93-99

2. Trouillas J, Roy P, Sturm N, Dantony E, Cortet-Rudelli C, Viennet G, Bonneville JF, Assaker R, Auger C, Brue T, Cornelius A, Dufour H, Jouanneau E, Francois P, Galland F, Mougel F, Chapuis F, Villeneuve L, Maurage CA, Figarella-Branger D, Raverot G, members of, Barlier H, Bernier A, Bonnet M, Borson-Chazot F, Brassier F, Caulet-Maugendre G, Chabre S, Chanson O, Cottier P, Delemer JF, Delgrange B, Di Tommaso E, Eimer L, Gaillard S, Jan S, Girard M, Lapras JJ, Loiseau V, Passagia H, Patey JG, Penfornis M, Poirier A, Perrin JY, Tabarin GA (2013) A new prognostic clinicopathological classification of pituitary adenomas: a multicentric case-control study of 410 patients with 8 years post-operative follow-up. Acta Neuropathol 126(1), 123-135. https://doi.org/10.1007/s00401-013-1084-y

3. Vieira JO Jr, Cukiert A, Liberman B (2006) Evaluation of magnetic resonance imaging criteria for cavernous sinus invasion in patients with pituitary adenomas: logistic regression analysis and correlation with surgical findings. Surg Neurol 65(2):130-135. https://doi.org/10.1016/j.surneu.2005.05.021 (discussion 135)

4. Kitano M, Taneda M, Shimono T, Nakao Y (2008) Extended transsphenoidal approach for surgical management of pituitary adenomas invading the cavernous sinus. J Neurosurg 108(1):2636. https://doi.org/10.3171/JNS/2008/108/01/0026

5. Micko AS, Wohrer A, Wolfsberger S, Knosp E (2015) Invasion of the cavernous sinus space in pituitary adenomas: endoscopic verification and its correlation with an MRI-based classification. J Neurosurg 122(4):803-811. https://doi.org/10.3171/2014.12. JNS141083

6. Nishioka H, Fukuhara N, Horiguchi K, Yamada S (2014) Aggressive transsphenoidal resection of tumors invading the cavernous sinus in patients with acromegaly: predictive factors, strategies, and outcomes. J Neurosurg 121(3):505-510. https://doi. org/10.3171/2014.3.JNS132214

7. Yamada S, Inoshita N, Fukuhara N, Yamaguchi-Okada M, Nishioka H, Takeshita A, Suzuki H, Ito J, Takeuchi Y (2015) Therapeutic outcomes in patients undergoing surgery after diagnosis of Cushing's disease: a single-center study. Endocr J 62(12):11151125. https://doi.org/10.1507/endocrj.15-0463

8. Hofstetter CP, Shin BJ, Mubita L, Huang C, Anand VK, Boockvar JA, Schwartz TH (2011) Endoscopic endonasal transsphenoidal surgery for functional pituitary adenomas. Neurosurg Focus 30(4):E10. https://doi.org/10.3171/2011.1.FOCUS10317

9. Micko A, Oberndorfer J, Weninger WJ, Vila G, Hoftberger R, Wolfsberger S, Knosp E (2019) Challenging Knosp high-grade pituitary adenomas. J Neurosurg.https://doi. org/10.3171/2019.3.JNS19367

10. Charalampaki P, Reisch R, Ayad A, Welschehold S, Conrad J, Wuster C (2006) Image-guided endonasal transsphenoidal microsurgical treatment of recurrent microadenomas of the pituitary gland. Minim Invasive Neurosurg 49(2):93-97. https://doi. org/10.1055/s-2006-932170

11. Elias WJ, Chadduck JB, Alden TD, Laws ER Jr (1999) Frameless stereotaxy for transsphenoidal surgery. Neurosurgery 45(2):271275 (discussion 275-277)

12. Enchev Y (2009) Neuronavigation: geneology, reality, and prospects. Neurosurg Focus 27(3):E11. https://doi. org/10.3171/2009.6.FOCUS09109

13. Gong J, Mohr G, Vezina JL (2007) Endoscopic pituitary surgery with and without image guidance: an experimental comparison. Surg Neurol 67(6):572-578. https://doi.org/10.1016/j.surne u.2006.08.083 (discussion 578)

14. Kajiwara K, Nishizaki T, Ohmoto Y, Nomura S, Suzuki M (2003) Image-guided transsphenoidal surgery for pituitary lesions using Mehrkoordinaten Manipulator (MKM) navigation system. Minim Invasive Neurosurg 46(2):78-81. https://doi. org/10.1055/s-2003-39340

15. Lasio G, Ferroli P, Felisati G, Broggi G (2002) Image-guided endoscopic transnasal removal of recurrent pituitary adenomas. Neurosurgery 51(1):132-136 (discussion 136-137)

16. McCutcheon IE, Kitagawa RS, Demasi PF, Law BK, Friend KE (2004) Frameless stereotactic navigation in transsphenoidal surgery: comparison with fluoroscopy. Stereotact Funct Neurosurg 82(1):43-48. https://doi.org/10.1159/000076660

17. McGrath BM, Maloney WJ, Wolfsberger S, Hill R, Massoud E, Imran SA, Clarke DB (2010) Carotid artery visualization during anterior skull base surgery: a novel protocol for neuronavigation. Pituitary 13(3):215-222. https://doi.org/10.1007/s1110 2-010-0220-0 
18. Thomale UW, Stover JF, Unterberg AW (2005) The use of neuronavigation in transnasal transsphenoidal pituitary surgery. Zentralbl Neurochir 66(3):126-132. https://doi. org/10.1055/s-2005-836602 (discussion 132)

19. Zhao Y, Yu S, Wang R, Zhao J (2006) Clinical application of a neuronavigation system in transsphenoidal surgery of pituitary macroadenoma. Neurosurg Rev 29(4):306-311. https://doi. org/10.1007/s 10143-006-0031-y (discussion 311-302)

20. Micko A, Hosmann A, Wurzer A, Maschke S, Marik W, Knosp E (2019) Wolfsberger S An advanced protocol for intraoperative visualization of sinunasal structures: experiences from pituitary surgery. J Neurosurg.https://doi.org/10.3171/2019.3.JNS1985

21. Lang M, Silva D, Dai L, Kshettry VR, Woodard TD, Sindwani $R$, Recinos PF (2018) Superiority of constructive interference in steady-state MRI sequencing over T1-weighted MRI sequencing for evaluating cavernous sinus invasion by pituitary macroadenomas. J Neurosurg.https://doi.org/10.3171/2017.9.JNS171699

22. Braileanu M, Hu R, Hoch MJ, Mullins ME, Ioachimescu AG, Oyesiku NM, Pappy A IInd, Saindane AM (2019) Pre-operative MRI predictors of hormonal remission status post pituitary adenoma resection. Clin Imaging 55:29-34. https://doi.org/10.1016/j. clinimag.2019.01.020

23. Davis MA, Castillo M (2013) Evaluation of the pituitary gland using magnetic resonance imaging: T1-weighted vs. VIBE imaging. Neuroradiol J 26(3):297-300. https://doi.org/10.1177/19714 0091302600307

24. Grober Y, Grober H, Wintermark M, Jane JA, Oldfield EH (2018) Comparison of MRI techniques for detecting microadenomas in Cushing's disease. J Neurosurg 128(4):1051-1057. https://doi. org/10.3171/2017.3.JNS163122

25. Blitz AM, Macedo LL, Chonka ZD, Ilica AT, Choudhri AF, Gallia GL, Aygun N (2014) High-resolution CISS MR imaging with and without contrast for evaluation of the upper cranial nerves: segmental anatomy and selected pathologic conditions of the cisternal through extraforaminal segments. Neuroimaging Clin N Am 24(1):17-34. https://doi.org/10.1016/j.nic.2013.03.021

26. Yagi A, Sato N, Taketomi A, Nakajima T, Morita H, Koyama Y, Aoki J, Endo K (2005) Normal cranial nerves in the cavernous sinuses:contrast-enhanced three-dimensional constructive interference in the steady state MR imaging. AJNR Am J Neuroradiol 26(4):946-950

27. Hisanaga S, Kakeda S, Yamamoto J, Watanabe K, Moriya J, Nagata T, Fujino Y, Kondo H, Nishizawa S, Korogi Y (2017) Pituitary macroadenoma and visual impairment: postoperative outcome prediction with contrast-enhanced FIESTA. AJNR Am J Neuroradiol 38(11):2067-2072. https://doi.org/10.3174/ajnr. A5394

28. Watanabe K, Kakeda S, Yamamoto J, Watanabe R, Nishimura J, Ohnari N, Nishizawa S, Korogi Y (2012) Delineation of optic nerves and chiasm in close proximity to large suprasellar tumors with contrast-enhanced FIESTA MR imaging. Radiology 264(3):852-858. https://doi.org/10.1148/radiol.12111363

29. Yamamoto J, Kakeda S, Shimajiri S, Takahashi M, Watanabe K, Kai Y, Moriya J, Korogi Y, Nishizawa S (2014) Tumor consistency of pituitary macroadenomas:predictive analysis on the basis of imaging features with contrast-enhanced 3D FIESTA at 3T. AJNR Am J Neuroradiol 35(2):297-303. https://doi.org/10.3174/ ajnr.A3667
30. Mete O, Lopes MB (2017) Overview of the 2017 WHO classification of pituitary tumors. Endocr Pathol 28(3):228-243. https://doi. org/10.1007/s12022-017-9498-z

31. Mert A, Micko A, Donat M, Maringer M, Buehler K, Sutherland GR, Knosp E, Wolfsberger S (2014) An advanced navigation protocol for endoscopic transsphenoidal surgery. World Neurosurg 82(6 Suppl):95-105. https://doi.org/10.1016/j.wneu.2014.07.032

32. Sanmillan JL, Torres-Diaz A, Sanchez-Fernandez JJ, Lau R, Ciller C, Puyalto P, Gabarros A (2017) Radiologic predictors for extent of resection in pituitary adenoma surgery. a single-center study. World Neurosurg 108:436-446. https://doi.org/10.1016/j. wneu.2017.09.017

33. Xie T, Zhang XB, Yun H, Hu F, Yu Y, Gu Y (2011) 3D-FIESTA MR images are useful in the evaluation of the endoscopic expanded endonasal approach for midline skull-base lesions. Acta Neurochir (Wien) 153(1):12-18. https://doi.org/10.1007/ s00701-010-0852-x

34. Grams AE, Kraff O, Kalkmann J, Orzada S, Maderwald S, Ladd ME, Forsting M, Gizewski ER (2013) Magnetic resonance imaging of cranial nerves at $7 \mathrm{~T}$. Clin Neuroradiol 23(1):17-23. https ://doi.org/10.1007/s00062-012-0144-3

35. Amemiya S, Aoki S, Ohtomo K (2009) Cranial nerve assessment in cavernous sinus tumors with contrast-enhanced 3D fast-imaging employing steady-state acquisition MR imaging. Neuroradiology 51(7):467-470. https://doi.org/10.1007/s00234-009-0513-z

36. Batista D, Courkoutsakis NA, Oldfield EH, Griffin KJ, Keil M, Patronas NJ, Stratakis CA (2005) Detection of adrenocorticotropin-secreting pituitary adenomas by magnetic resonance imaging in children and adolescents with cushing disease. J Clin Endocrinol Metab 90(9):5134-5140. https://doi.org/10.1210/ jc. 2004-1778

37. Chowdhury IN, Sinaii N, Oldfield EH, Patronas N, Nieman LK (2010) A change in pituitary magnetic resonance imaging protocol detects ACTH-secreting tumours in patients with previously negative results. Clin Endocrinol (Oxf) 72(4):502-506. https://doi.org /10.1111/j.1365-2265.2009.03646.x

38. Kasaliwal R, Sankhe SS, Lila AR, Budyal SR, Jagtap VS, Sarathi V, Kakade H, Bandgar T, Menon PS, Shah NS (2013) Volume interpolated 3D-spoiled gradient echo sequence is better than dynamic contrast spin echo sequence for MRI detection of corticotropin secreting pituitary microadenomas. Clin Endocrinol (Oxf) 78(6):825-830. https://doi.org/10.1111/cen.12069

39. Patronas N, Bulakbasi N, Stratakis CA, Lafferty A, Oldfield EH, Doppman J, Nieman LK (2003) Spoiled gradient recalled acquisition in the steady state technique is superior to conventional postcontrast spin echo technique for magnetic resonance imaging detection of adrenocorticotropin-secreting pituitary tumors. $\mathbf{J}$ Clin Endocrinol Metab 88(4):1565-1569. https://doi.org/10.1210/ jc. $2002-021438$

Publisher's Note Springer Nature remains neutral with regard to jurisdictional claims in published maps and institutional affiliations. 